\title{
Stroke Volume with Dynamic Cardiomyoplasty during Ventricular Fibrillation in the Acute Dog
}

\author{
Leslic Alexander Geddes, M.E., Ph.D.,* \\ Wolfgang JanAs, B.Sc., * James Cook B.S.E., ** \\ Marvin Hinds Ph.D., *** \\ and Stephen Francis BadYLAK M.D., Ph.D.*
}

\begin{abstract}
SUMMARY
The objective of this study was to determine the pumping capability of dynamic cardiomyoplasty during induced ventricular fibrillation. In this acutc study of 6 dogs, the pumping capability of the unconditioned left latissimus dorsi (LD) muscle (141 to $292 \mathrm{gm}$ ), wrapped around both ventricles, was investigated during induced ventricular fibrillation. Left-ventricular and femoral artery pressure, the ECG and aortic root flow velocity were monitored. Prior to inducing ventricular fibrillation, the ability of the unconditioned LD muscle to augment stroke volume (SV), was quantified as the area under the aortic flowvelocity record. The ventricles were then fibrillated and, after $10 \mathrm{sec}$, rhythmic $250 \mathrm{msec}$ trains $(1 / \mathrm{sec})$ of stimuli $(40 / \mathrm{sec})$ were delivered to the thoracodorsal nerve to contract the LD muscle tetanically. In no case could dynamic cardiomyoplasty produce the same SV as when the ventricles were beating normally. In one animal, the SV attained two percent of the normal SV by 5 contractions; in another, the SV reached one percent by 25 contractions. In the remaining animals, the SV varied around $20 \%$ of the prefibrillation SV. By 90 contractions, the stroke volume was $10 \%$ of the prefibrillation value. The progressive decrease in $\mathrm{SV}$ was likely a consequence of $\mathrm{LD}$ muscle ischemia and fatigue, since the latissimus dorsi muscle provided low blood flow during the period of fibrillation. (Jpn Heart J 35: 73-80, 1994)
\end{abstract}

Key words: Dynamic cardiomyoplasty

$\mathrm{D}$

YNAMIC cardiomyoplasty (CMP) is designed to provide cardiac assistance by synchronous tetanic contraction of a skeletal muscle wrapped around the ventricles. Since Kantrowitz et $\mathrm{al}^{1)}$ and Nakamura et $\mathrm{al}^{2\rangle}$ wrapped the canine

\footnotetext{
From the *Hillenbrand Biomedical Engineering Center, Purdue University, W. Lafayette, IN 47907-1293, **Undergraduate Biomedical Engineering Student, Purdue University, W. Lafayette, IN 47907, and ***Professor of Biology, Indiana Wesleyan University, Marion, IN 46953, USA.

Supported by grant HL 45883, National Heart, Lung and Blood Institute, Bethesda, MD and a grant from the Trask Fund, Purdue University, W. Lafayette, IN 47907, USA.

Received for publication July 16, 1993.

Accepted September 17, 1993.
} 
hemidiaphragm around the ventricles and made it contract during ventricular systole, experimental animal studies ${ }^{3-5)}$ have shown that cardiac output can be augmented by varying amounts up to $20 \%$. There is one report ${ }^{6 j}$ that describes dynamic CMP in the dog during ventricular fibrillation and states that an arterial pressure of $80 \mathrm{mmHg}$ was obtained 15-20 min after the onset of ventricular fibrillation. Another report ${ }^{7}$ showed that very little blood flow was produced when the ventricles were fibrillating and the encircling skeletal muscle was contracted rhythmically. The present study was undertaken to determine the SVpumping capability of the unconditioned left $\mathrm{LD}$ muscle wrapped around the canine heart during a worst-case condition, electrically induced ventricular fibrillation.

\section{Methods and Materials}

All studies described herein were performed in accordance with the Guide for the Care and Use of Laboratory Animals, published by the U.S. Department of Health, Education, and Welfare (DHHS publication No. NIH 85-23, revised 1985). This study was approved by the Purdue University Animal Care and Use Committee.

Six dogs, ranging in weight from 21 to $32 \mathrm{~kg}$ were anesthetized with sodium pentothal $(10 \mathrm{mg} / \mathrm{kg}$, iv), intubated and placed in right-lateral recumbency. Anesthesia was maintained with Isofluorane, $1.5 \%$ with oxygen. The animals were supported with positive-pressure ventilation and by the administration of fluids and sodium bicarbonate, based on repeated acid-base and electrolyte determinations. A cephalic vein catheter was inserted for the administration of fluids and drugs. A femoral artery catheter was introduced to collect blood samples and monitor abdominal aortic pressure. Cardiopulmonary bypass was not used.

Surgical procedure: An incision was made from the caudal axilla to the midpoint of the last rib along the ventral border of the left latissimus dorsi muscle. Intercostal vascular branches, extending to the ventral and dorsal borders of the muscle, were isolated and divided. The caudal (inferior) origin of the latissimus dorsi muscle and the dorsal (posterior) fascial origin attachments were freed. The thoracodorsal artery and vein and a branch of the lateral thoracic artery remained the only blood supply to the isolated LD muscle. The insertion of the muscle to the proximal humerus was transected and moved to the adjacent second rib so that when the muscle was contracted tetanically, there was no force applied to the limb, thereby preventing stretching the thoracodorsal artery, vein and nerve trunk. A bipolar stimulating electrode was then placed against the nerve and covered by a plastic sleeve. One-third to one-half of the third rib was 
removed to permit translocation of the LD muscle into the thorax. The lateral incision site was then closed. The animal was repositioned dorsally and a median sternotomy was performed. The root of the ascending aorta was dissected from the pulmonary artery and an ultrasonic flow probe (Transonics, Ithaca, NY) was placed to monitor aortic-root flow velocity (AFV). Stroke volume was determined by measuring the area under each calibrated AFV wave. A pigtail catheter was inserted through the left atrial appendage and advanced into the left ventricle to monitor left-ventricular pressure without compromising the aortic valve.

The left LD muscle (weight range: 141 to $292 \mathrm{gm}$ ), was wrapped around the heart, with the pericardium intact, in a clockwise direction and sutured to the pericardium so that the largest amount of ventricular musculature was encompassed. The ventricles were wrapped tightly enough to avoid constriction and to allow the encircling muscle to experience the intraventricular pressure. Tactile sensation and a very slight transient reduction in left-ventricular systolic pressure were used to guide the wrapping tightness. A bipolar sensing electrode was placed on an atrial appendage to detect the $\mathbf{P}$ wave.

Pulse generator: The trains of tetanic stimuli $(250 \mathrm{msec})$ were delivered to the motor nerve by a stimulus isolation unit driven by a Grass stimulator (Grass Instruments, Quincy, MA). This stimulator generated each train of tetanic stimuli (40/sec, 0.1 msec pulses). A custom-built timing circuit gated the stimulator. When the heart was in sinus rhythm, the timing circuit was triggered by the $\mathrm{P}$ wave such that the train of tetanic stimuli began $70 \mathrm{msec}$ after the peak of the $R$ wave. The timing circuit also permitted delivery of a train of stimuli for each $P$ wave or a submultiple thereof, i.e. 1:2 to 1:10. An output of $4 \mathrm{~V}$ was adequate to excite all of the axons in the thoracodorsal nerve. When the ventricles were fibrillated, the delay circuit was disabled and rhythmic 250-msec trains of stimuli were delivered to the thoracodorsal nerve at one train/sec.

Experimental study: Control records were obtained of the cardiac electrogram, which contained the $\mathrm{R}$ wave, left-ventricular pressure (LVP), pulsatile aortic-root flow velocity (AFV) and femoral artery pressure. With sinus rhythm, a train duration of $250 \mathrm{msec}$ was delivered $70 \mathrm{msec}$ after the peak of the $\mathrm{R}$ wave to determine the ability of the LD muscle to augment LVP, AFV (from which SV was determined) and femoral artery pressure. The ventricles were then fibrillated by delivering stimuli of $100 / \mathrm{sec}$ ( 2 msec pulse duration) to the ventricles. After about 10 seconds of fibrillation, the thoracodorsal nerve was stimulated rhythmically once per second.

\section{Results}

Figure 1 illustrates the cardiac electrogram, LVP, AFV and femoral artery pressure in a typical animal with normal sinus rhythm. The delivery of a single 
train of stimuli to the LD muscle (identified by A), augmented LVP, AFV and femoral artery pressure. Measurement of the areas under the AFV curves for the assisted (A) and preassisted beat revealed that stroke volume was augmented by $15 \%$.

Figure 2 illustrates the cardiac electrogram, LVP, AFV and femoral artery pressure prior to and following the induction of ventricular fibrillation. After 10 sec of fibrillation, $250 \mathrm{msec}$ trains of stimuli were delivered to the thoracodorsal nerve to contract the LD muscle once per second. The record in Figure 2 is for the animal shown in Figure 1. Although there were reasonably large-amplitude pulses in the LVP record, the AFV pulses were very small in amplitude. The LVP pulses disappeared in about 70 seconds.

\section{CARDLAC ELECTROGRAM}
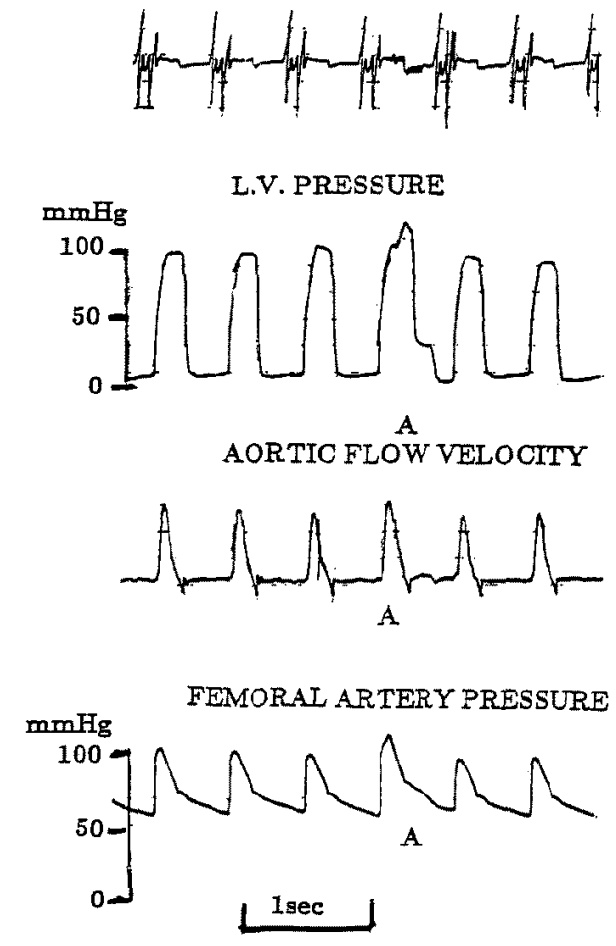

Figure 1. Cardiac electrogram, showing the $P$ and $R$ waves, left-ventricular pressure (LVP), aortic flow velocity (AFV) and femoral artery pressure. When the latissimus muscle was contracted to assist the ventricles (A), there was an augmentation in LVP, AFV and femoral artery pressure. Note that the AFV for the assisted beat (A) is taller and broader than that for the preassisted beat, indicating a $15 \%$ augmentation in stroke volume. Note also that the systolic LVP and femoral artery pressures are slightly less for the post-assisted beat, indicating a slight ventricular overemptying by the assisted beat $(\mathrm{A})$. 

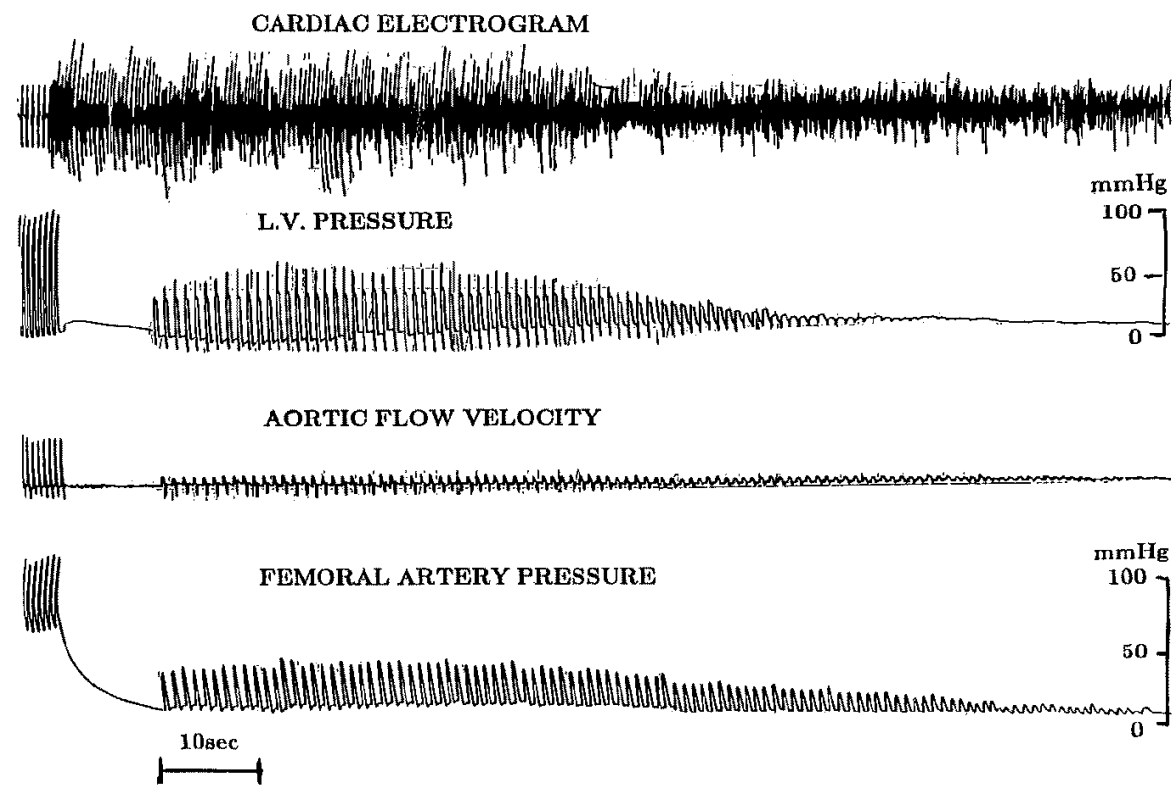

Figure 2. Cardiac electrogram, left-ventricular (LV) pressure, aortic flow velocity (AFV) and femoral artery pressure during normal sinus rhythm (left) and immediately after the precipitation of ventricular fibrillation. Ten seconds after the onset of fibrillation, the ventricles were compressed rhythmically with $250 \mathrm{msec}$ trains of tetanic muscle contractions. These data are from the animal shown in Figure 1.

Figure 3A illustrates the stroke volumes for each animal as a function of the number of muscle contractions. The stroke volumes are shown as the percent of the stroke volume for the last beat just before inducing ventricular fibrillation. Figure $3 \mathrm{~B}$ presents the average percent stroke volume versus $\mathrm{LD}$ contraction number for all of the animals. In no case was the first dynamic CMP contraction able to produce the same SV as was produced by the heart during sinus rhythm. In one animal the percent SV decreased to two percent in 5 contractions. In another animal the percent SV fell to one percent in 25 contractions. In the other animals, the average stroke volume varied around $20 \%$ as the LD muscle continued to be contracted rhythmically. After about 90 contractions, the stroke volume was $10 \%$ of the control value.

\section{Discussion}

The results of this study are inconsistent with those reported by Termet et al $^{6)}$ and consistent with those of Anderson et al. ${ }^{7)}$ After about 80 contractions, which represents about one and one-third minutes, stroke volume fell below $20 \%$ of the control value. However, there were variations in the percentage of stroke volume during rhythmic LD muscle contraction. Recall that the atria were beat- 

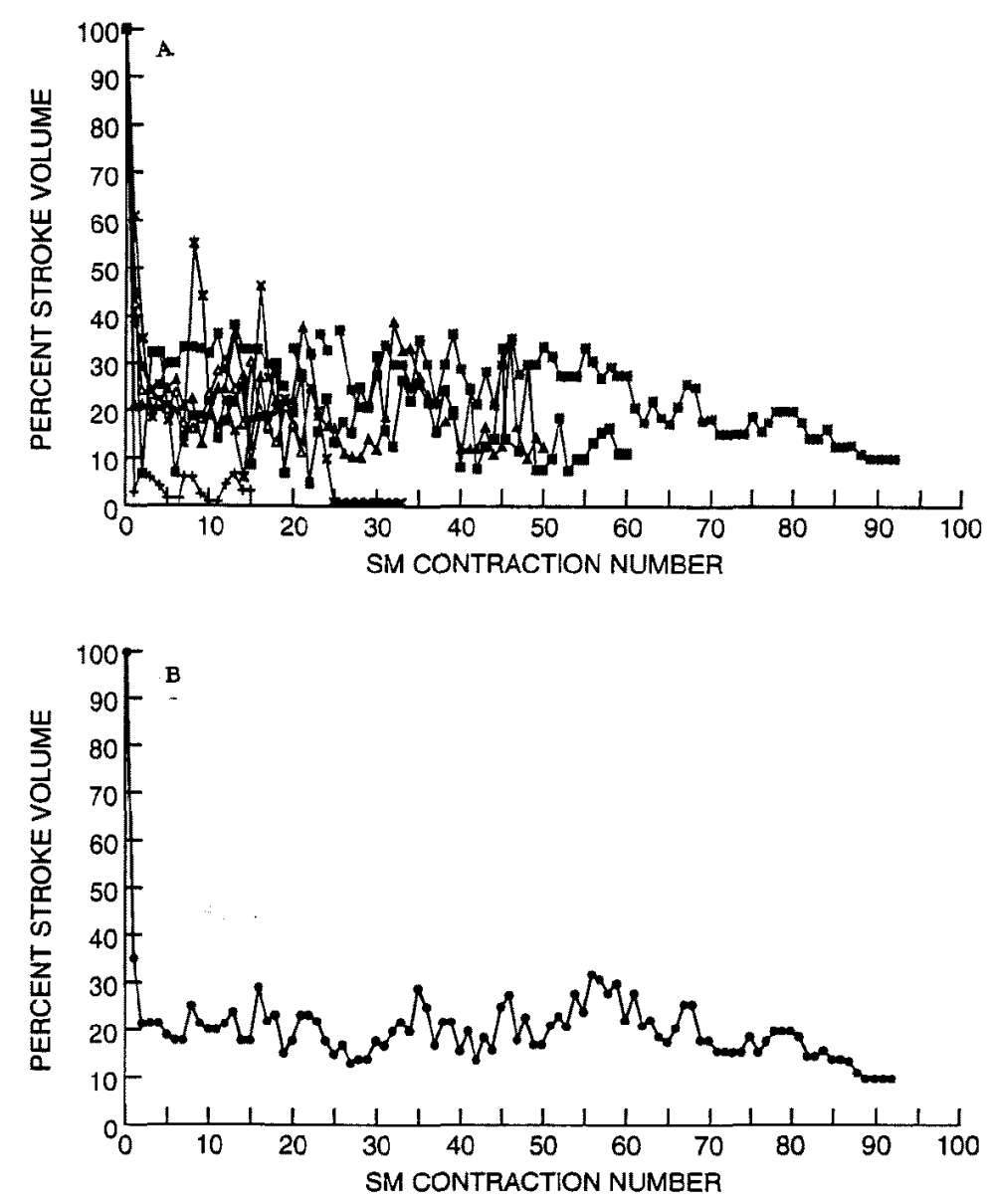

Figure 3. Percent stroke volume versus $L D$ contraction number before and during ventricular fibrillation (A) for each animal. Contraction number zero represents the percent stroke volume for the last beat before initiating ventricular fibrillation. In B the average stroke volume versus contraction number for all of the animals is shown.

ing spontaneously and the autonomic nervous system was probably trying to increase peripheral resistance. It is likely that both contributed to the variations in SV amplitude. In one animal, SV dropped immediately to a few percent of the prefibrillation SV. This was not due to any technical difficulty. We speculate that the muscle wrap was not as tight in this animal.

The reduced stroke volume of dynamic CMP during ventricular fibrillation clearly reduced blood flow to the LD muscle. Thus, with prolonged pumping, the LD muscle became ischemic and failed. However, this is not the case with dynamic CMP when the muscle assists ventricular systole.

The force of skeletal muscle contraction depends on the preload. For the maximum force of contraction with a muscle-wrapped pouch, the pressure 
therein must be in the order of arterial pressure, ${ }^{8,9)}$ as predicted by the FrankStarling law. In the dynamic CMP role, the intraventricular pressure available to preload the encircling skeletal muscle is much less, being left-ventricular, enddiastolic pressure (LVEDP). Therefore the force of contraction is much less than maximal. During ventricular fibrillation, the preload may be less than LVEDP because the compliance of the fibrillating ventricles is less than that of normal ventricles, thereby reducing the ability of the skeletal muscle to propel blood. It is worthy of note that open-chest manual cardiac compression during ventricular fibrillation can provide a cardiac output of about one half of that when the ventricles are beating normally. ${ }^{10)}$ However, in this case, the preload on the muscles of the rescuer's hand does not determine the force of ventricular compression.

With dynamic GMP, the tightness of the muscle wrap and the intraventricular pressure both affect the force of skeletal muscle contraction. The muscle wrap must be sufficiently tight to both experience the intraventricular pressure and decrease ventricular diameter during nerve stimulation. A wrap that is too tight will produce cardiac constriction and ischemia in both the myocardium and skeletal muscle. A wrap that is too loose will fail to provide preload and fail to communicate the contraction shortening to decrease ventricular size. We believe that the differences in SV among the animals may be due to differences in muscle-wrap tightness. As yet there has been no investigation of the effect of muscle-wrap tightness on stroke volume with dynamic CMP.

In summary, dynamic CMP was unable to restore stroke volume to its prefibrillation level. The average SV after about three LD muscle contractions was about $20 \%$ of the prefibrillation stroke volume. If the pumping ratio is less than $1 / 1$, the augmentation in cardiac output would be less than $20 \%$. However, in no case was this percentage sustained beyond 80 contractions.

\section{References}

1. Kantrowitz A, McKinnon MP: The experimental use of the diaphragm as an auxiliary myocardium. Surg Forum 9: 265, 1959

2. Nakamura K, Glenn WWL: Graft of the diaphragm as a functioning substitute for the myocardium. J Surg Res. 4: 435, 1964

3. Chachques JC, Grandjean P, Schwartz K, Miheileanu S, Fardeau M, Swynghedauw B, Fontaliran F, Romero $N$, Wisnewsky $C$, Perier $\mathbf{P}$ : Effect of latissimus dorsi dynamic cardiomyoplasty on ventricular function. Circulation 78 (Suppl III): 203, 1988

4. Kao RL, Christlieb IY, Magovern GJ, Park SB, Magovern GJ, Jr: The importance of skeletal muscle fiber orientation for dynamic cardiomyoplasty. J Thorac Cardiovasc Surg 99: 134, 1990

5. Lucas CMHB, Van der Veen FH, Cheriex EC, Larusso R, Havenith M, Penn OCKM, Wellans HIJ: Long-term follow-up after dynamic cardiomyoplasty in goats. PACE 15: 1430, 1992

6. Termet $H_{3}$ Chalencon JL, Estour E, Galland P, Faure JP: Transplantation sur le myocarde $\mathrm{d}^{2}$ un muscle strie excite par pacemaker. Ann Chir Thor Cardiov 5: 260, 1966

7. Anderson WA, Andersen JS, Acker MA, Hammond RL, Chin AJ, Douglas PS, Khalafalla AS, Salmons 
S, Stephenson LW: Skeletal muscle grafts applied to the heart; a word of caution. Circulation 78 (Suppl III): $11-180,111-190,1988$

8. Spotnitz HM, Merker C, Malm JR: Applied physiology of the canine rectus abdominis. Trans ASAIO 20: 747,1974

9. Geddes LA, Badylak SF, Wessale JL, Tacker, WA: The use of electrically stimulated skeletal muscle to pump blood. PACE 13: 344, 1990

10. Del Guercio LRM, Feins NR, Cohn JD, Coomaraswamy RP, Wollman SB, State D: Comparison of blood flow during external and internal cardiac massage in man. Circulation 31 and 32 (Suppl I): I 171, 1965 